\title{
Benefícios da hemisferectomia em pacientes com hemimegalencefalia
}

\author{
Helder José Lessa Zambelli*, José Jorge Facure**, Concezio Di Rocco***, \\ Aldo lannelli***, Donizeti Cesar Honorato***** \\ Disciplina de Neurocirurgia da UNICAMP (Universidade Estadual de Campinas). Seção de Neurocirurgia Pediátrica \\ do Instituto de Neurocirurgia da Universidade Católica de Roma
}

\begin{abstract}
RESUMO
A hemisferectomia é realizada em muitos casos de hemimegalencefalia para controlar a epilepsia refratária ao tratamento medicamentoso. No entanto, apesar de haver concordância sobre a efetividade desse procedimento para controle das crises, a escolha da técnica cirúrgica utilizada ainda é motivo de debate.

Relatamos os casos de três crianças com hemimegalencefalia submetidas à hemisferectomia para tratamento de suas crises refratárias: uma foi submetida à hemisferectomia anatômica e as outras duas, à hemisferectomia funcional.

Os resultados confirmam a efetividade da hemisferectomia para o controle das crises epilépticas nesses casos.
\end{abstract}

PALAVRAS-CHAVE

Hemimegalencefalia. Hemisferectomia anatômica. Hemisferectomia funcional.

\begin{abstract}
Hemispherectomy for hemimegalencephaly

Hemispherectomy is required in the most cases of hemimegalencephaly to control epilepsy refractory to medical treatment. Although there is a general agreement on the effectiveness of the procedure in controlling the seizure disorder, the choice of the surgical technique is still a subject of debate. A series of three children with hemimegalencephaly who had undergone anatomical hemispherectomy (one case) and functional hemispherectomy (two cases) is reported.

The results obtained confirmed the effectiveness of the procedure to control the seizures due to hemimegalencephaly.

\section{KEYWORDS}

Hemimegalencephaly. Anatomical hemispherectomy. Functional hemispherectomy.
\end{abstract}

\section{Introdução}

A hemimegalencefalia é uma rara anomalia do desenvolvimento do sistema nervoso reportado pela primeira vez, em 1835, por Sims ${ }^{32}$, caracterizada por crescimento de um hemisfério cerebral com dilatação e configuração irregular do seu ventrículo, com graus variáveis de displasia cortical, incluindo alteração das camadas corticais, polimicrogiria multifocal, áreas de lissencefalia, ectopia da substância cinzenta e anormalidades vasculares ${ }^{6,45}$.

Clinicamente, a hemimegalencefalia é caracterizada por epilepsia, hemiparesia, hemianopsia e, em grande parte dos casos, por retardo psicomotor severo ${ }^{15}$, podendo ocorrer isoladamente ou em associação com anomalias da pele e do corpo, como o nevo sebáceo linear (síndrome de Jadassohn) ${ }^{4}$, a hipomelanose de Ito $^{10}$, o angioma cutâneo similar aos encontrados em pacientes com doença de Sturge -Weber e o gigantismo unilateral usualmente do lado do hemisfério afetado ${ }^{13}$. A hemisferectomia é o tratamento cirúrgico indicado aos pacientes com hemimegalencefalia e, geralmente, resulta em drástica redução da freqüência ou completo desaparecimento das crises epilépticas. Quando realizada precocemente, a cirurgia parece favorecer o desenvolvimento psicomotor ${ }^{13,15,20,34}$.

\footnotetext{
*Neurocirurgião do Hospital das Clínicas da UNICAMP e Pós-Graduando da FCM - UNICAMP.

** Professor Livre-Docente em Neurocirurgia.

***Professor Diretor Chefe da Seção de Neurocirurgia Pediátrica do Instituto de Neurocirurgia da Universidade Católica de Roma.

****Professor Doutor da Seção de Neurocirurgia Pediátrica do Instituto de Neurocirurgia da Universidade Católica de Roma.

*****Professor Chefe da Disciplina de Neurocirurgia da UNICAMP.
} 
A alta incidência de complicações, verificada em mais de um terço dos $\operatorname{casos}^{18,28,30}$, justifica as modificações técnicas no procedimento com o objetivo de reduzir o risco cirúrgico, minimizando a retirada de tecido cerebral e maximizando a desconexão funcional do parênquima cerebral anormal deixado in loc $u^{112,31,42}$.

No presente trabalho, relatamos os benefícios observados em três crianças com hemimegalencefalia que foram submetidas à hemisferectomia por apresentarem epilepsia intratável, sendo realizados diferentes tipos de procedimentos cirúrgicos. A hemisferectomia anatômica extratalamocaudada foi realizada em um paciente e, nos outros dois, foi realizada a hemisferectomia funcional de acordo com Rasmussen ${ }^{30}$.

\section{Casuística e métodos}

Três crianças, com idades de 6 meses, 3 anos e 8 anos, portadoras de hemimegalencefalia, foram submetidas à hemisferectomia por apresentarem epilepsia refratária ao tratamento medicamentoso. Das três crianças, uma menina de 8 anos apresentava somente hemimegalencefalia. As outras duas apresentavam outras malformações: o menino de 3 anos apresentava a síndrome de Jadassohn (nevo sebáceo linear) e a menina de 6 meses, hemigigantismo facial direito e hemangioma facial. Nos três pacientes, o hemisfério direito foi o afetado. O diagnóstico incluiu o exame clínico, o morfológico (tomografia computadorizada de crânio, ressonância nuclear magnética) e a eletroencefalografia em todos os casos; o SPECT (single photon emission computed tomography) foi realizado em apenas um caso. Uma criança foi submetida à hemisferectomia anatômica extratalamocaudada e as outras duas, submetidas à hemisferectomia funcional de Rasmussen. Em todos os pacientes foi utilizada uma drenagem subdural temporária para remover resíduos de sangue da cavidade cirúrgica.

Os pacientes foram acompanhados no pós-operatório com tomografia computadorizada, ressonância nuclear magnética, eletroencefalografia de repetição e análise da evolução neurológica e neuropsicológica.

\section{Resultados}

O primeiro paciente de 8 anos de idade, sexo feminino, apresentava crises convulsivas tônico-clônicas generalizadas e crises parciais complexas com uma freqüência que superava a 30 crises diárias e com grande resistência ao tratamento medicamentoso (altas doses de fenobarbital, carbamazepina e lamotrigina). Ao exame neurológico, notava-se uma tetraparesia severa, com predomínio à esquerda, e retardo no deenvolvimento neuropsicomotor. No eletroencefalograma, evidenciouse decréscimo da atividade elétrica na região frontal direita ou da atividade rítmica na freqüência $7 \mathrm{~Hz}$ a $8 \mathrm{~Hz}$ composta de espículas com reversão de fase em F4 - F8, propagando-se para a região homóloga contralateral e, depois, para as demais regiões; o traçado interictal revelou espículas multifocais e um alentecimento difuso da atividade de base. A telemetria registrou 59 crises clinicoeletrográficas durante período pouco superior a cinco horas. Na tomografia computadorizada e ressonância nuclear magnética de crânio, observou-se aumento no volume de todo o lobo frontal direito e ínsula, englobando o córtex e substância branca, estendendo-se à radiação do corpo caloso, preservando os núcleos da base; além disso, havia alargamento do corpo caudado, espessamento cortical e apagamento de alguns sulcos, deformidade do sistema ventricular à direita e, na ressonância magnética, marcado alongamento T1 e T2 da substância branca (não-mielinização). A cintilografia de perfusão cerebral (SPECT) mostrou uma área focal de hiperperfusão na região frontoparietal direita e na porção anterior do giro do cíngulo com sinais indiretos de dilatação ventricular à direita, mais intensa na porção anterior, sugerindo alta probabilidade de foco epileptogênico na região frontoparietal direita e no giro do cíngulo. Essa criança foi submetida à hemisferectomia funcional direita no Hospital das Clínicas da UNICAMP (Figura 1), teve meningite pós-operatória, necessitando de limpeza da cavidade cirúrgica com colocação de drenagem subdural transitória e apresentou algumas crises durante o sono no pós-operatório imediato, mas permaneceu sem crises durante um ano, com uso de fenitoína e fenobarbital; após um ano, passou a apresentar raras crises acordada e abalos rítmicos em membro superior direito que desaparecem após revisão do medicamento. A criança está sendo acompanhada há 3 anos e meio do ponto de vista clínico e por imagem, estando sem crises em uso de medicação, apresentandose inalterada quanto à hemiparesia esquerda e à hemianopsia, com ganhos no aspecto neuropsicomotor, conseguindo comer sozinha. Eletroencefalografias seriadas durante o seguimento mostraram distúrbio epileptiforme em região temporal posterior direita e parietoccipital direita e assimetria com diminuição da atividade elétrica no hemisfério direito. A cintilografia de perfusão cerebral (SPECT) no pós-operatório tardio mostrou ausência de perfusão cortical em grande extensão do hemisfério cerebral direito, notando-se apenas pequena área de acentuada hipoperfusão no córtex occipital à direita com perfusão normal no córtex cerebral esquerdo. 

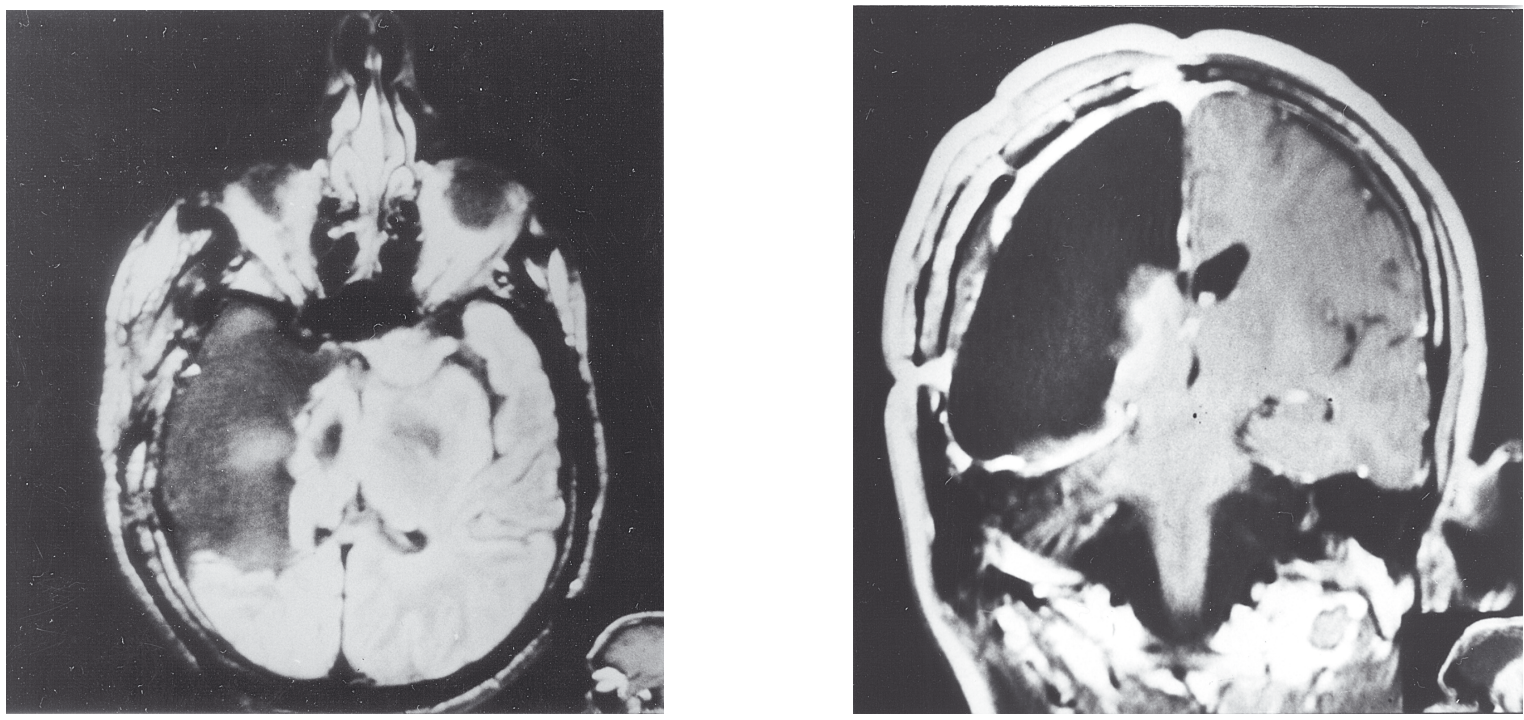

Figura 1 - Ressonância magnética pós-operatória de paciente submetido à hemisferectomia funcional.

O segundo paciente, de 3 anos de idade, sexo masculino, apresentava crises parciais complexas e crises convulsivas tônico-clônicas generalizadas não diárias. Ao exame, notava-se presença de nevo sebáceo linear acompanhado de hemiparesia à esquerda e grave retardo no desenvolvimento neuropsicomotor. Fazia uso de ACTH, clobazam e valproato de sódio. A eletroencefalografia evidenciou atividade elétrica cerebral do tipo contínuo e rítmico composta por espículas em todo o hemisfério direito. Na tomografia computadorizada e na ressonância nuclear magnética de crânio observou-se aumento no volume de todo o hemisfério direito com espesamento cortical e apagamento de sulcos e deformidade do sistema ventricular à direita. A criança foi submetida à hemisferectomia anatômica à direita, na seção de Neurocirurgia Pediátrica da Universidade Católica de Roma, apresentando febre e distonia transitória no pós-operatório imediato, bem como a necessidade de limpeza da cavidade cirúrgica e colocação de drenagem subdural transitória. O paciente foi seguido por um ano no pósoperatório, permanecendo sem crises, sem uso de medicação, com melhora da hemiparesia esquerda e ganhos no desenvolvimento neuropsicomotor.

O terceiro paciente, de 6 meses de idade, sexo feminino, apresentava crises parciais complexas com generalização secundária reentrantes, com alta freqüência diária, mesmo em uso de altas doses de valproato, vigabatrina e luminalete. Ao exame, notavase gigantismo hemifacial à direita, acompanhado da presença de hemangioma facial à direita, hemiparesia à esquerda e moderado retardo no desenvolvimento neuropsicomotor. A eletroencefalografia evidenciou presença de burst suppression hemisférico direito, de elevada voltagem; já no hemisfério esquerdo, a atividade elétrica cerebral era do tipo contínuo, constituída de ritmo misto, observando-se difusão da atividade epileptiforme hemisférica direita sobre regiões do hemisfério contralateral, sendo utilizada telemetria para avaliação clínica das crises. $\mathrm{Na}$ tomografia computadorizada de crânio observou-se um aumento do volume de todo o hemisfério direito, com espessamento cortical e apagamento de sulcos com deformidade do sistema ventricular à direita; já na ressonância nuclear magnética, notou-se aumento dos tecidos moles da hemiface direita - de sinal hiperintenso em T1 e T2, compatível com malformação lipomatosa de tecidos moles - , aumento do volume do hemisfério direito com dilatação do ventrículo lateral direito, principalmente o corno temporal, hipertrofia da substância branca profunda do hemisfério direito e deformidade dos sulcos, com alteração do sinal em T1 e T2 na substância branca do lobo frontal direito. A criança foi submetida à hemisferectomia funcional direita, na seção de Neurocirurgia Pediátrica da Universidade Católica de Roma, necessitando de colocação da drenagem subdural transitória. O paciente foi seguido por um ano e meio, no pós-operatório, permanecendo sem crises em uso de medicação com melhora da hemiparesia esquerda e ganhos no desenvolvimento neuropsicomotor.

\section{Discussão}

Os resultados da hemisferectomia em pacientes com epilepsia refratária ao tratamento medicamentoso e conseqüente à má-formação do desenvolvimento 
cortical têm mostrado melhora satisfatória na freqüência das crises, segundo a literatura ${ }^{8,13,17}$. King e cols. ${ }^{22}$, em 1985 , relataram o caso de uma criança com hemimegalencefalia, que, após hemisferectomia, não teve mais crises; foi o primeiro relato da literatura de melhora do prognóstico de crises em pacientes com hemimegalencefalia após tratamento cirúrgico, sugerindo que a hemisferectomia precoce seria o tratamento de escolha para essa condição clínica. Em 1989, Di Rocco e cols. ${ }^{36}$ publicaram dois casos de hemisferectomia em crianças com hemimegalencefalia, com completa remissão das crises e melhora no desenvolvimento neuropsicomotor. Em 1990, Vigevano e cols. ${ }^{37}$ publicaram trabalho em que defendem a efetividade da hemisferectomia em pacientes com epilepsia intratável e hemimegalencefalia. Koelfen e cols. ${ }^{23}$, em 1993, relataram um caso de hemimegalencefalia, com crises intratáveis e encefalopatia severa, que foi submetido à hemisferectomia aos 9 meses de idade para preservar as funções do hemisfério cerebral contralateral. Palmini e cols. ${ }^{29}$ defenderam, num trabalho de 1994, que a hemisferectomia pode eliminar ou diminuir significativamente a freqüência das crises em pacientes com hemimegalencefalia e epilepsia intratável com medicação, dependendo da localização, grau e extensão das anormalidades histopatológicas. Em 1994, foi publicada, por Di Rocco e cols. ${ }^{14}$, uma série de 13 pacientes que foram submetidos à hemisferectomia extratalamocaudada, apresentando hemimegalencefalia e crises intratáveis, com uma drástica redução na freqüência e severidade das crises em 12 dos 13 pacientes. Chen e cols. ${ }^{11}$ publicaram, em 1994, dois casos de crianças com hemimegalencefalia submetidas à hemisferectomia funcional, com grande declínio na freqüência das crises e óbvio ganho no desenvolvimento neuropsicomotor nesses pacientes, defendendo que a hemisferectomia funcional deve ser realizada o mais precoce possível para controle das crises. Em 1995, Mathis e cols. ${ }^{26}$ relataram o caso de um paciente com hemimegalencefalia e epilepsia intratável, no qual uma "hemisferectomia embólica" pré-operatória permitiu que o paciente permanecesse um ano sem crises após a embolização; além disso, ressaltaram que esse procedimento foi útil na subseqüente hemisferectomia, limitando a perda de sangue durante a cirurgia. Wyllie e cols. ${ }^{44}$, em artigo sobre cirurgia de epilepsia em crianças, publicado em 1996, concordam com as conclusões prévias da literatura de que, em casos bem selecionados, incluindo hemimegalencefalia, a cirurgia traz benefícios ao tratamento de epilepsia de difícil controle. Zupanc ${ }^{47}$, em 1996, num artigo sobre epilepsia em pacientes pediátricos, defendeu a importância da hemisferectomia precoce em pacientes com epilepsia e hemimegalencefalia. Também, Humberclaude e cols. ${ }^{20}$, em 1997, escreveram sobre a importância da hemisferectomia funcional precoce, relatando o caso de um menino com hemimegalencefalia e epilepsia catastrófica, totalmente controlado das crises e com melhora no desenvolvimento neuropsicomotor, após três anos de cirurgia, preservando o desenvolvimento das funções corticais do hemisfério contralateral. A hemisferectomia funcional modificada foi relatada por Yoshioka e cols. ${ }^{46}$, em 1999, apresentando o caso de uma criança livre das crises alguns dias após a cirurgia. Em 1999, Sugimoto e cols. ${ }^{33}$ defenderam que o prognóstico das crises em pacientes submetidos à hemisferectomia é excelente se comparado com aqueles submetidos à ressecção cortical focal. Battaglia e cols. ${ }^{3}$, em 1999, publicaram um artigo sobre o prognóstico do desenvolvimento neurocognitivo e da epilepsia em crianças com hemimegalencefalia, tratadas cirurgicamente com hemisferectomia, concluindo que houve uma melhora significativa das crises, com prognóstico cognitivo menos favorável, apesar de ser notada uma melhora na competência cognitiva em todos os casos, e que os déficits neurológicos não pioram após a cirurgia; quanto melhor o desenvolvimento cognitivo antes da intervenção cirúrgica, menores as alterações morfológicas detectadas nos exames de neuroimagem e maior a integridade anatômica e funcional do hemisfério não acometido, parece ser melhor o prognóstico cognitivo ${ }^{3}$. Maehara e cols. ${ }^{25}$ descreveram, em 2000, outro paciente com hemimegalencefalia tratado com hemisferectomia funcional, com melhora tanto do ponto de vista de crises convulsivas quanto do desenvolvimento neuropsicomotor. Cinco de seis pacientes com hemimegalencefalia, relatados por Carreno e cols. ${ }^{8}$, em 2001, apresentaram melhora marcante das crises após serem submetidos à hemisferectomia funcional; portanto, tanto na hemimegalencefalia quanto nos outros tipos de má-formações do desenvolvimento cortical, existe melhora no prognóstico das crises em pacientes submetidos à hemisferectomia ${ }^{17}$.

Em um dos três pacientes da nossa casuística, a hemimegalencefalia ocorreu isoladamente; nos outros dois, essa má-formação ocorreu em associação com assimetria facial direita (lipomatose facial), acompanhada de angioma facial em uma das crianças e presença de nevo sebáceo linear em outra criança. A lipomatose congênita da face associada à hemimegalencefalia ipsilateral é extremamente rara $^{2,13}$; já a síndrome do nevo sebáceo linear é uma facomatose caracterizada, em geral, por uma tríade constituída por nevo sebáceo linear de Jadassohn, epilepsia e retardo mental $1^{5,7,13,16,17,19,24,33}$, podendo ocorrer outros sintomas neurológicos, oftalmológicos, esqueléticos, urogenitais e cardiovasculares; anormalidades estruturais do cérebro e crânio são observadas freqüentemente ( $72 \%$ dos casos), sendo mais 
comuns a dilatação de um dos ventrículos laterais, a hemimegalencefalia e a hemimegacrania ${ }^{35}$.

Os resultados obtidos nos três pacientes da nossa casuística confirmam a efetividade da hemisferectomia, independentemente da técnica utilizada, no controle das crises de pacientes com hemimegalencefalia. Nas três crianças, as crises convulsivas desapareceram. Uma criança apresentou distonia transitória por um certo período de tempo,conforme relatado por outros $^{13,43}$. Sabe-se atualmente que pacientes com más-formações hemisféricas do desenvolvimento cortical, particularmente a hemimegalencefalia, apresentam uma melhora significativa no que diz respeito às crises convulsivas após a hemisferectomia, ficando livres das crises ou com melhora marcante na freqüência das destas ${ }^{8,9,21,38,42}$. Os diversos procedimentos cirúrgicos utilizados para hemisferectomia são considerados igualmente eficazes no controle das crises, existindo uma tendência atual a favor da hemisferectomia funcional ${ }^{8,12,31}$, já que, aparentemente, a hemisferectomia anatômica apresenta maior número de complicações ${ }^{12,31}$, como hemossiderose e hidrocefalia secundária ${ }^{13,40,41}$. Os três pacientes operados não apresentaram hidrocefalia.

Os trabalhos sobre hemimegalencefalia indicam uma incidência relativamente alta de hidrocefalia associada a esta má-formação ${ }^{27,34}$; já, a hidrocefalia pós-operatória parece ser maior quando são utilizados procedimentos clássicos como a hemisferectomia anatômica se comparados com os chamados procedimentos cirúrgicos modificados como a hemisferectomia funcional na qual a hidrocefalia pós-operatória aparece em torno de $10 \%$ dos $\operatorname{casos}^{13,21,31,38,39,42}$.

A hemossiderose é a complicação mais preocupante relacionada com a hemisferectomia anatômica, considerando-se que a mortalidade tardia na hemisferectomia era depedente dos efeitos da deposição de $\mathrm{Fe}^{+2}$ no parênquina cerebral por repetidas hemorragias intracranianas e pelo desenvolvimento de hidrocefalia não detectada, antes da introdução da tomografia. No entanto, os trabalhos considerando hemossiderose são praticamente ausentes na literatura após os anos $70^{13}$.

\section{Referências}

1. ADAMS CBT: Hemispherectomy: A modification. J Neurol Neurosurg Psychiatry 46:617-9, 1993.

2. AYDINGOZ U, EMIR S, KARLI-OGUZ K, KOSE G, BUYUKPAMUKCU M: Congenital infiltrating lipomatoses of the face with ipsilateral hemimegalencephaly. Pedriat Radiol 32:106-9, 2002

3. BATTAGLIA D, DI ROCCO C, IUVONE L, ACQUAFONDATA C, IANNELLI A, LETTORI D, GUZZETTA F: Neurocognitive development and epilepsy outcome in children with surgically treated hemimegalencephaly. Neuropediatrics 30:307-13, 1999.

4. BIANCHINE JW: The nevus sebaceous of Jadassohn. Am J Dis Child 120:223-8, 1970.

5. BONIOLI EV, BERTOLA A, DI STEFANO A, BELLINI C: Sebaceous nevus syndrome: report of two cases. Pediatr Neurol 17:77-9, 1997.

6. BOSMAN C, BOLDRINI R, DIMITRI L, DI ROCCO C, CORSI A: Hemimegalencephaly. Hystological, immunohystochemical ultrastructural and citofluorimetric study of six patients. Childs Nerv Syst 12:76575, 1996.

7. BRODSKY MC, KINCANNON JM, NELSON ADESERAN P, BROWN HH: Oculocerebral dysgenesis in the linear nevus sebaceous syndrome. Ophthalmology 104:497-503, 1997.

8. CARRENO M, WYLLIE E, BINGAMAN W, KOTAGAL P, COMAIR Y, RUGGIERI P: Serzure outcome after functional hemispherectomy for malformations of cortical development. Neurology 57:331-3, 2000.

9. CARSON BS, JAVEDON SP, FREEMAN JM, VINING EPG, ZUCHERBERGER AL, LAUER JA, GUARNIERI M: Hemispherectomy: A hemidecortication approach and review of 52 cases. J Neurosurg 84:903-11, 1996.

10. CAVENAGH EC, HART BL, ROSE D: Association of linear sebaceous nevus syndrome and unilateral megalencephaly. AJNR 14:405-8, 1996.

11. CHEN HL, WANG PS, TU YK, TSENG SH, YAO YT, YOUNG C, SHEN YZ: Hemimegalencephaly treated by hemispherectomy: report of two cases. J Formos Med Assoc 93:961-66, 1994.

12. DELALANDE O, PINARD JM, BASDEVAN C: Hemispherotomy: A new procedure for central disconnection. Epilepsia 33(suppl 3):99-100, 1992.

13. DI ROCCO, IANNELLI A: Hemimegalencephaly and intractable epilepsy: complications of hemispherectomy and their correlations with the surgical te crnique. A report on 15 cases. Pediatr Neurosurg 33:198-207, 2000.

14. DI ROCCO C, IANNELLI A, MARCHESE E, VIGEVANO F, ROSSI GF: Surgical treatment of epileptogenic hemimegalencephaly. Minerva Pediatr 46:231-7, 1994.

15. DI ROCCO C, IANNELLI A, MARCHESE E, VIGEVANO F, ROSSI GF: Terapia chirurgica dell' emimegalencefalia epiliptogena. Minerva Pediatr 46:231-7, 1994.

16. DOGE NN, DOBYNS WB: Agenesis of the corpus callosum and Dandy-Walker malformation associated with hemimegalencephaly in the sebaceous nevus syndrome. Am J Med Genet 56:147-50, 1995.

17. EDWARDS JC, WYLLIE E, RUGGERI PM, BINGAMAN W, LUDERS H, KOTA, DINNER DS, MORRIS HH, PRAYSON RA, COMAIR YG: Seizure outcome after surgery for epilepsy due malformation of cortical development. Neurolgy 55:1110-4, 2000.

18. FALCONER MA, WILSON PJE: Complications related to delayed hemorrhage after hemispherectomy. J Neurosurg 30:413-26, 1969.

19. HERMAN TE, SIEGEL MJ: Hemimegalencephaly and linear nevus sebaceous syndrom. Perinatol 21:3368, 2001.

20. HUMBERTOCLAUDE VT, COUBES PA, ROBAIN O, ECHEME BB: Early hemispherectomy in a case of hemimegalencephaly. Pediatr Neurosurg 27:268-71, 1997.

21. KANEV PM, FOLEY CM, MILES D: Ultrasound-tailored functional hemispherectomy for surgical control of seizures in children. J Neurosurg 86:762-7, 1997. 
22. KING M, STEPHERSON JB, ZIERVOGEL M, DOYLE D, GALBRAITH S: Hemimegalencephaly a case for hemispherectomy? Neuropediatrics 16:46-55, 1985.

23. KOELFEN W, FREUND M, RONR H, SCHULTZE C, HOLTHAUSEN H: Hemimagalencephaly. Therapy with hemispherectomy. Monatsschr Kinderleilkd 141:300-2, 1993.

24. KRUSE B, POUWELS PJ, CHRISTEN HJ, FRAHM J, HANEFELD FA: Proton magnetic resonance spectroscopy of linear nevus sebaceus syndrome. Pediatr Neurol 18:176-80, 1998.

25. MAEHARA T, SHIMIZU H, SHIGETOMO R, TAMAGAWA K: Functional hemispherectomy for children aged 2 years or less for the treatment of intractable epilepsy caused by cortical dysgenesis. No To Hattatsu 32:395-400, 2000.

26. MATHIS JM, BAN JD, ALBRINGHT AL, HORTON JA: Hemimegalencephaly and intractable epilepsy treated with embolic hemispherectomy. AJNR 16:1076-9, 1995.

27. MYLES ST, DARWISH H, HAMILTON MG, LANGE VL: Complications of surgical management of hemimegalencephaly in childhood. Childs Nerv Syst 14:679-82, 1998.

28. OPPENHEIMER DR, GRIFEITH HB: Persistent intracranial bleeding as a complication of hemispherectomy. $\mathrm{J}$ Neurol Neurosurg Psychiatry 9:229-40, 1966.

29. PALMINI A, GAMBARDELLA A, ANDERMANN F, DUBEAU F, DA COSTA JC, OLIVIER A, TAMPIERI D, ROBITALLE Y, PAGLIOLI E, PAGLIOLI NETO: Operative strategies for patients with cortical dysplastic lesions and intractable epilepsy. Epilepsia 35(suppl 6): 557-71, 1994.

30. RASMUSSEN T: Hemispherectomy for seizures revisited. Can J Neurol Sci 10:71-8, 1983.

31. SCHRAMM J, BEHRENS E, ENTZIAN W: Hemispherical deaferentatron: An alternative to functional hemispherectomy. Neurosurgery 36:509-16, 1983.

32. SIMS JM: On hypertrophy and atrophy of the brain. Medicochir Trans 19:315-80, 1835.

33. SUGIMOTO T, OTSUBO H. HWANG PA, HOFFMAN HJ, JAY V, SNEAD OC: Outcome of epilepsy surgery in the first three years of life. Epilepsia 40:560-5, 1999.

34. TAHA JM, CRONE KR, BERGER TS: The role of hemispherectomy in the treatment of holohemispheric hemimegalencephaly. J Neurosurg 81:37-42, 1994.

35. VAN DE WARRENBURG BP, VAN GULIK S, RENIER WO, LAMMENS M, DOELMAN JC: The linear naevus sebaceus syndrome. Clin Neurol Neurosurg 100:12632, 1998
36. VIGEVANO F, BERTINI E, BOLDRINI R, BOSMAN C, CLAPS D, DI CAPUA M, DI ROCCO C, ROSSI GF: Hemimegalencephaly and intractable epilepsy: benefits of hemispherectomy. Epilepsia 30:833-43, 1989.

37. VIGEVANO F, DI ROCCO C: Effectiveness of hemispherectomy in hemimegalencephaly with intractable Seizures. Neuropediatrics 21:222-3, 1990.

38. VILLEMURE JD, ADAMS CBT, HOFFMAN JH, PEACOCK WJ: Hemispherectomy. In Engel J (ed): Surgical Treatment of Epilepsies. New York, Raven Press, 1993, pp 511-8.

39. VILLEMURE JG: Cerebral hemispherectomy for epilepsy. In SchmideK HH, Sweet WH (eds): Operative Neurosurgical Techniques. Philadelphia, Saunders, 1995, pp 1341-50.

40. WHITE HH: Corebral hemispherectomy in the treatment of infantile hemiplegia. Review of the literature and report of two cases. Confinia Neurol 21:1-50, 1961.

41. WILSON PJE: Cerebral hemspheretomy for infantile hemiplegia. A report of 50 cases. Brain 93:147-10, 1970.

42. WINSTON KR, WELCH K, ADLER JR, ERBA G: Cerebral hemicorticectomy for epilepsy. J Neurosurg 77:889-95, 1992

43. WROE S, RICHENS A, COMPSTON A: Bilateral balistic movements occuring as a late conplication of hemispherectomy and responding to sulpiride. J Neurol 233:315-6, 1986.

44. WYLLIE E, COMAIR YG, KOTAGAL P, RAJA S, RUGGIERI P: Epilepsy surgery in infants. Epilepsia 37:625-37, 1996.

45. YASHA TC, SANTOS HV, SHANKAN SK: Hemimegalencephaly - Morphological and immunocytochemical study. Appl Neuropathol 16:17-22, 1997.

46. YOSHIOKA H, HASEGAWA K, SAKAMOTO T, KITHARA M, SHIMIZU H: Modfied functional hemispherectomy in hemimegalencephaly. Brain Dev 21:125-8, 1999.

47. ZUPANC ML: Update on epilepsy in pediatric patients. Mayo Clin Proc 71:899-916, 1996.

Original recebido em abril de 2002

Aceito para publicação em setembro de 2002

\section{Endereço para correspondência:}

Helder José Lessa Zambelli

Rua Roberto Gomes Pedrosa, 314

CEP 13095-120 - Campinas, SP 